\title{
Prevalence and risk factors of domestic violence in women attending the National Guard Primary Health Care Centers in the Western Region, Saudi Arabia, 2018
}

R. Wali ${ }^{1,2,3^{*}}$ (D, A. Khali i, ${ }^{1,3}$, R. Alattas ${ }^{2,3}$, R. Foudah ${ }^{2,3}$, I. Meftah ${ }^{2,3}$ and S. Sarhan ${ }^{2,3}$

\begin{abstract}
Background: Domestic violence (DV) is considered a public health issue in Saudi Arabia as well as a violation of a fundamental human right. DV causes many acute and chronic physical and mental health consequences. Cultural taboos and lack of awareness regarding the appropriate support services can increase the number of cases annually. The objective of the study was to assess the prevalence and risk factors of DV in women attending the National Guard Primary Health Care Clinics in the Western Region of Saudi Arabia.

Methods: A cross-sectional study was conducted with patients attending five Primary Health Care Centers in Jeddah from August 2017 to February 2018. A convenient sampling method was used. In total, 1845 participants were invited to complete a self-report validated Arabic version of the Norvold Domestic Abuse Questionnaire (NORAQ) to determine the prevalence and risk factors of DV. All women between 18 and 65 years who met the inclusion criteria were included. The data were analyzed using SPSS (Statistical Package Social Sciences) version 24.0.

Results: The lifetime prevalence of DV in the study sample was 33.24\%, with psychological abuse the most prevalent (48.47\%), followed by physical abuse (34.77\%) and sexual abuse (16.75\%). A small proportion (4.1\%) suffered from all three types of abuse. Risk factors for being a victim of abuse include being single or divorced, having a postgraduate level of education, employed, and being financially independent of the husband.

Conclusion: DV is prevalent in Saudi Arabia. Modernization has shifted the risk factors, identifying the risk factors and victim characteristics would support the development and implementation of preventive and screening programs to facilitate the early identification of cases as well as the initiation of empowerment programs for Saudi women.
\end{abstract}

Keywords: Domestic violence, Women abuse, Primary health care, Prevalence, Risk factors, Saudi Arabia

\section{Summary Boxes}

What is already known on this subject?

Domestic violence is prevalent in Saudi Arabia. Cultural Taboos play an essential role in the occurrence of abuse. All types of Domestic Violence are prevalent in Saudi Arabia, and women are reluctant to disclose being abused due to several factors, one of which is saving the family from destruction and feeling ashamed. For the last 2 years, with

\footnotetext{
* Correspondence: dr_razazwali@hotmail.com

${ }^{1}$ Ministry of National Guard-Health Affairs, Jeddah, Saudi Arabia

${ }^{2}$ King Abdullah International Medical Research Center, Jeddah, Saudi Arabia

Full list of author information is available at the end of the article
}

the new Saudi Vision (2030), different taboos have been changed, and women are more empowered and able to decide on her decisions and well-being.

\section{What does this study add?}

This study is the first study that demonstrated that risk factors for domestic violence could change with the change in population demographics as well as with modernization. It is essential to know about the current risk factors of DV to address proper educational, screening, and preventive programs. This study also demonstrates that, with the change of the DV risk factors, the prevalence of domestic violence remains the same, 
which lets us think of developing interventions that aim to decrease the prevalence like public educational programs and screening programs for women presenting to the primary care centers.

\section{Background}

Domestic Violence (DV) is defined as violent behavior perpetrated by a member of a family against another to humiliate, dominate, and govern the person's behavior [1-3]. Violent behavior has many forms, including physical (direct or indirect), sexual, psychological, or emotional [2]. Although some societies do not recognize DV as a social issue, it is a global phenomenon as it breaches fundamental human rights [4].

DV against women has become a global problem and is prevalent in both Western and Arab countries. As reported by the World Health Organization (WHO), 29.8\% of women in America and 25.4\% in the European regions have been victims of physical and/or sexual abuse by a partner or sexual abuse by a non-partner [5]. In addition, 1 in 3 women in Palestine, Egypt, Tunisia, and Israel was domestically abused in 2003-2005 [6, 7]. Globally, approximately one- third of women experienced DV. Societies that do not recognize DV as a problem may overlook the physical, emotional, and social consequences of the victims, their families, and society as a whole, with suicide attempts being the most recent consequence $[4,8]$.

Saudi Arabia is predominantly a men-dominated culture, and men may exhibit controlling behavior towards women. There are many cultural taboos within Saudi families, and a woman is responsible for taking care of her family and preventing family destruction, even if she is unhappy in the relationship. These taboos may underpin why women avoid asking for assistance or report abuse to her family or doctors. Recently, the role of women in the community has been a topic of discussion, and DV against Saudi women has been highlighted. In 2019, several cases have been reported of Saudi women fleeing the country due to being physically or emotionally abused [9]. At present, DV is recognized as a public health and human right concern in Saudi society.

Several studies have been conducted in Jeddah, Al Madinah, Al-Ahsa, and Riyadh in Saudi Arabia, to estimate the prevalence of DV [10-13] In AL Madinah in 2012, the prevalence of ever-abused women was $34 \%$ [10]. In the Eastern Region of Saudi Arabia, Al-Ahsa, the lifetime prevalence of DV against women ranged from 39.3 to $57.7 \%$ in 2010 [11]. In Jeddah, the prevalence was estimated to be $34 \%$ [12]. In Riyadh, Saudi married women had a lifetime prevalence of $43.0 \%$ for any type of violence [13].

The scope of this dilemma has not been investigated in the National Guard population in the Western Region of Saudi Arabia. The study aimed to determine the prevalence and risk factors of DV in women and attendees of the National Guard Primary Health Care clinics in the Western Region of Saudi Arabia. This study could serve as the foundation for further studies and culture-specific screening programs in the future.

\section{Methods}

This research was an across-sectional study conducted from 2017 to 2018, in the National Guard Primary Healthcare Centers in the Western Region. The centers include Iskan, Bahra, the Specialized Polyclinic in Jeddah, Alsharea clinic in Makkah, and Iskan clinic in Taif. All are satellite clinics of King Abdulaziz Medical City (KAMC).

The sample size was calculated at a $95 \%$ confidence interval (CI) level with a 50\% response distribution and a margin of error of $\pm 5 \%$. The required sample size was determined to be 1845 using Raosoft software (http://www.raosoft.com/samplesize.html).

A non-probability convenient sampling technique was used to select study participants. Women patients and attendees, age 18 to 65 years, were invited to complete a self-administered questionnaire. Trained data collectors interviewed participants who could not read or write. Critically ill women were excluded from enrollment.

The validated Arabic version of the Norvold Domestic Abuse Questionnaire (NORAQ) was used. It is a questionnaire that can be used to study the prevalence of DV in addition to the risk factor. It also highlights different types of abuse, severity, and perception of victims about their health. The Arabic version was also validated and made it more useful in collecting data about sensitive issues. The Arabic NORAQ total content validity index is 0.90 , while the alpha reliability coefficients were 0.75 for the total scale and ranged from 0.75 to 0.77 for the subscales.

The questionnaire measures three types of abuse: psychological, physical, and sexual [14].

The questionnaire had five sections. The first section included questions regarding the participant's general health status as well as her socio-demographic characteristics, including age, level of education, religion, marital status, and age when married, and if married, whether she was divorced, or widowed. Also included were questions related to the participant's employment status, household income, and financial dependence on her husband. The participant's health status was explored in terms of the frequency of hospital visits and admissions in the past year, as well as any somatic symptoms or symptoms of depression or insomnia.

The second, third, and fourth sections consisted of questions regarding emotional, physical, and sexual abuse. The participants were primarily asked about a history of abuse. If DV occurred, she was asked to continue to answer more questions that are detailed. The questions focused on the age she was initially abused, abuse within the last year, the extent of the impact of 
the abuse on the patient's life, the identity of the abuser, and if the abuser was abusing substances. Besides, disclosure and assistance seeking behavior were explored. The last part of the questionnaire explored the patient's attempts to receive assistance, awareness of the institutes offering support services, and being fearful and at risk of being abused.

Ethical approval for the study was obtained from the Institutional Review Board (IRB) of King Abdullah International Medical Research Center (KAIMRC). Ethical principles were maintained throughout the research process. All participants signed informed consent, and they were informed about their right to withdraw from the study. Confidentiality and anonymity were assured. The data were stored in workplace computers with access to only the researchers. The investigators were available to answer any questions about the questionnaire during data collection.

The data were analyzed using SPSS (Statistical Package Social Sciences) version 24.0. Continuous variables are presented as mean and standard deviation and categorical variables as frequency and percentage. For inferential statistics, Pearson's Chi-square was used to test the relation between DV and the qualitative independent variables. The $\mathrm{T}$-Test was used to test the relationship between DV and the quantitative independent variables. A $p$-value $<0.05$ was considered significant.

\section{Results}

\section{Demographic characteristics}

The sample size realized as 1845 participants aged between 18 and 65 years. All were Saudi nationals, belonged to the Muslim religion and dependents of National Guard employees. The mean age of the sample was $32.24 \pm 10.92$ years. Married women constituted $71.96 \%(n=1304)$ of the sample, with the mean duration of marriage $1.80 \pm$ 1.15 years, and the mean age when married $16.87 \pm 9.44$ years. The proportion of single women were $22 \%(n=406)$ with smaller proportions of divorced women $2.5 \%$, $(n=$ 46) and being widowed 3.09\%, $(n=56)$ (Table 1).

In terms of accommodation, the majority of the study population $83.13 \%(n=1503)$, lived in the city with $15.76 \%(n=285)$ living in the village. More than half 59.86\% $(n=1081)$ owned their own home and $40.14 \%$ $(n=725)$ rented accommodation. The mean number of rooms per dwelling was $5.11 \pm 1.90$, and the mean number of family members $6.13 \pm 3.178$ (Table 1 ).

Regarding financial dependence on the caregiver $(n=1383)$, more than half $77.65 \%(n=1047)$ were financially dependent and a small proportion $22.34 \%(n=309)$ were financially independent. Where the majority $(64.58 \%, n=1165)$ receives monthly income more than $5000 \mathrm{SR}$, with a small proportion $35.42 \%$, $(n=639)$ receives less than 5000 SR. In terms of the educational level of the sample $(n=1808)$, a small proportion was illiterate $10.06 \%(n=182)$, undergraduate $45.66 \%(n=$
Table 1 Demographic characteristics of the sample

\begin{tabular}{|c|c|c|}
\hline a & & \\
\hline Variable & Mean & SD \\
\hline Age $(n=1810)$ & 32.246 & 10.927 \\
\hline Age at marrige $(n=1703)$ & 16.86 & 9.442 \\
\hline Duration of marriage $(n=1717)$ & 1.801 & 1.152 \\
\hline Family members $(n=1801)$ & 6.13 & 3.178 \\
\hline Number of rooms $(n=1700)$ & 5.111 & 1.9071 \\
\hline b & & \\
\hline Variable & Frequency & $\%$ \\
\hline Residence $(n=1845)$ & & \\
\hline - UK (unknown) & 20 & 1.11 \\
\hline - City & 1503 & 83.13 \\
\hline - Village & 285 & 15.76 \\
\hline Financially dependent $(n=1383)$ & & \\
\hline - Yes & 1074 & 77.65 \\
\hline - No & 309 & 22.34 \\
\hline Marital status $(n=1812)$ & & \\
\hline - Single & 406 & 22.41 \\
\hline - Married & 1304 & 71.96 \\
\hline - Divorce & 46 & 2.54 \\
\hline - Widow & 56 & 3.09 \\
\hline Education level $(n=1808)$ & & \\
\hline - Illiterate & 182 & 10.06 \\
\hline - Undergraduate & 826 & 45.66 \\
\hline - Graduate & 770 & 42.56 \\
\hline - Postgraduate & 31 & 1.71 \\
\hline Residence $(n=1806)$ & & \\
\hline - Owner & 1081 & 59.86 \\
\hline - Rent & 725 & 40.14 \\
\hline Employment status ( $n=1813$ ) & & \\
\hline - Student & 5 & 0.28 \\
\hline - Employed & 258 & 14.23 \\
\hline - Housewife & 1546 & 85.27 \\
\hline - Retired & 4 & 0.22 \\
\hline Income & & \\
\hline - less than 5000 & 639 & 35.42 \\
\hline - More than 5000 & 1165 & 64.58 \\
\hline
\end{tabular}

$826)$, graduate $42.56 \%(n=770)$ and postgraduate $1.71 \%(n=$ $31)$. The majority $(n=1813)$ were housewives $85.27 \%(n=$ 1546), with a small proportion employed $14.23 \%(n=258)$ and $0.22 \%(n=4)$ retired.

\section{Prevalence of domestic violence}

The lifetime prevalence of DV in the sample $(n=1802)$ was $33.24 \%(n=599)$ (Fig. 1). Psychological abuse was 
the most prevalent $48.47 \%(n=460)$ followed by physical abuse $34.77 \%(n=330)$ and a smaller proportion $16.75 \%$ $(n=159)$ reported sexual abuse. A small proportion $4.1 \%(n=75)$ reported suffering all three types of abuse. (Tables 2 and 3), (Fig. 2).

\section{The relationship between demographic characteristics and abuse status in the sample}

Table 4 displays a significant relationship between abuse status and level of education $(p<0.005)$. The abused status is most prevalent in participants with a postgraduate education $38.7 \%(n=12)$ followed by a graduate-level education 38.2\% $(n=292)$, undergraduate education 30.7\% $(n=252)$ and $23.5 \% \quad(n=42)$ were illiterate. Being employed was also statistically significant $(p<0.005)$. The prevalence of abuse was highest among employed women $45.9 \% \quad(n=118)$ compared to housewives or retired women. The place of residence, type of home, and family income were not statistically significant.

The social status was significantly associated with abuse, and most prevalent in the divorced group 63\%, $(n=29)$ followed by being single $39.1 \%(n=158)$, widowed $32.1 \%$ $(n=18)$, and lowest in the married group $30.4 \%(n=394)$. Being financially independent of the husband $41.7 \%$ ( $n=$ $129)$, was also statistically significant. The duration of marriage had no significant association with the abuse status.

\section{Psychological abused participant characteristics and source of abuse}

This study estimates the prevalence of psychological abuse at $48.47 \%(n=460)$. The mean age when first abused was $13.07 \pm 11.27$ years and the mean age of informing another person about being abused $14.86 \pm 11.63$ years. The mean score on a 10 point scale $(0=$ no suffering, $10=$ suffering
Table 2 Prevalence of Abuse in Sample

\begin{tabular}{lccllll}
\hline Prevalence of abuse & \multicolumn{2}{c}{ Abused } & & \multicolumn{2}{c}{ Not abused } \\
\cline { 2 - 3 } & $n$ & & & & & \\
\hline Ever abused $(n=1802)$ & 599 & 33.24 & & 1203 & 66.76 \\
All type of abuse $(n=1813)$ & 75 & 4.1 & & 1738 & 95.9 \\
\hline
\end{tabular}

terribly) for current suffering in the abused group was $5.34 \pm$ 2.98. About a third of the sample $33.92 \%(n=153)$ did not attempt to find assistance as they did not consider themselves to be suffering, $39.46 \%$ ( $n=178)$ did not attempt to find assistance though they were suffering and only a small proportion $26.6 \%(n=128)$ attempted to obtain assistance. (Table 5).

Of the psychologically abused group, $32.66 \%(n=147)$ were psychologically abused in the last year and the abuser was the current husband $16.27 \%(n=98)$, father $15.44 \%$ $(n=93)$, brother $13.62 \%(n=82)$, mother $8.63 \%(n=52)$, a person known by the family $7.14 \%(n=43)$, and friends $6.31 \%(n=38)$. According to participants, $37.73 \%(n=140)$ of the abusers were smokers, $4.58 \%(n=17)$ were drug users, and $2.156 \%(n=8)$ were alcoholics. (Table 5).

In terms of disclosure, more than half $55.29 \%(n=251)$ did not disclose the abuse at all, 30.84\% $(n=140)$ partially disclosed, and only $13.88 \%(n=63)$ disclosed the abuse to another person. Regarding disclosing to a medical practitioner, $84.78 \%(n=195)$ did not disclose at all, 2.17\% $(n=5)$ thought her doctor knew, 7.39\% $(n=17)$ disclosed only when the doctor inquired, and 5.65\% ( $n=$ 13) disclosed immediately. (Table 5).

\section{Physical abused patient characteristics and source of abuse}

The prevalence of physical abuse in this study was 330 (34.77\%). The mean age when first abused was $12.79 \pm$

\section{Prevalence Of Domestc Violence}

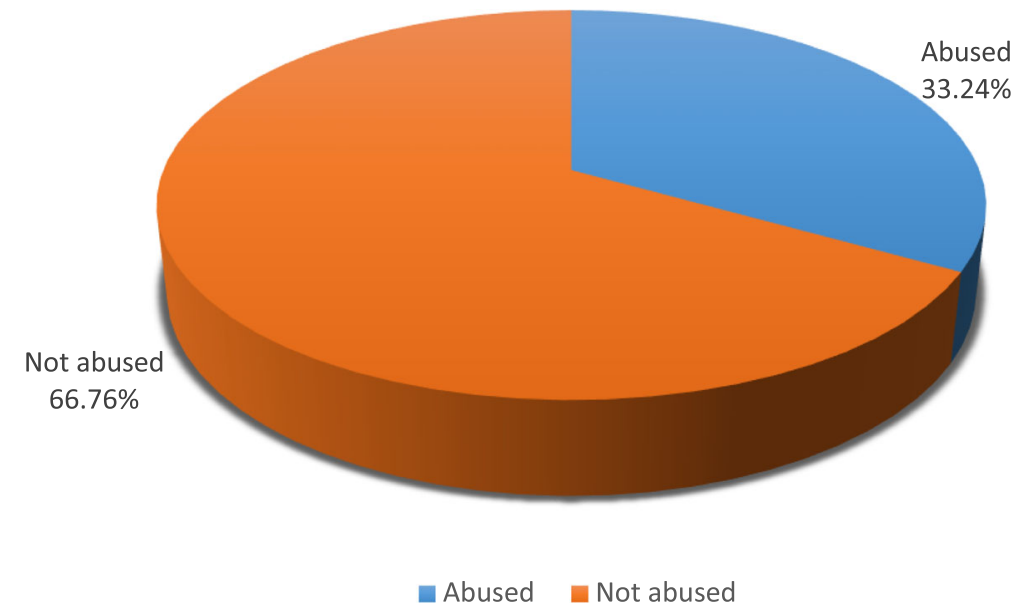

Fig. 1 Prevalence of Domestic Violence 
Table 3 Domestic Violence by Type

\begin{tabular}{lll}
\hline Type of & Abused & \\
\cline { 2 - 3 } abuse & $\mathrm{n}$ & $\%$ \\
\hline Psychological & 460 & 48.47 \\
Physical & 330 & 34.77 \\
Sexual & 159 & 16.75 \\
\hline
\end{tabular}

10.09 years, while the mean age when informing a person about being abused was $13.63 \pm 10.57$ years. The mean score on a10 point scale $(0=$ no suffering, $10=$ suffering terribly) for the current situation of the abused participants was $4.79 \pm 2.814$. (Table 6).

Of the physically abused group, $29.81 \%(n=96)$ were abused in the last year. Half of the physically abused women $50.77 \%(n=164)$ did not attempt to obtain assistance because they thought they were not suffering, $28.48 \%(n=92)$ did not attempt to be assisted though they were experiencing suffering and 20.74\% $(n=67)$ attempted to be assisted. (Table 6).

The source of abuse was the current husband $24.12 \%$ $(n=55)$, father and brother $17.54 \%(n=40)$ and $17.98 \%$ $(n=41)$ respectively, ex-husband $9.6 \%(n=22)$ and friends $6.14 \%(n=14)$. Some of the abusers were smokers $43.07 \%$ $(n=112)$, drug abusers 5\% $(n=13)$ and $3.46 \%(n=9)$ abused alcohol.

In terms of disclosure, $54.51 \%(n=175)$ did not disclose being abused, $30.52 \%(n=98)$ partially disclosed and only $14.95 \%(n=48)$ informed another person. Regarding informing their medical practitioner, $80.44 \%$ $(n=144)$ did not disclose, $1.67 \%(n=3)$ thought their doctor knew, $11.17 \%(n=20)$ disclose to the doctor when asked about abuse and 6.7\% $(n=12)$ immediately disclosed to her doctor. (Table 6).

\section{Sexual abused participants' characteristics and source of} abuse

The prevalence of sexual abuse was $16.75 \%(n=159)$. The mean age of the first experience of sexual abuse was $9.92 \pm 7.63$ years, and the mean age of disclosing the abuse was $11.92 \pm 8.49$ years. The mean score on a 10 point scale $(0=$ no suffering, $10=$ suffering terribly) for the current level of suffering $5.307 \pm 3.02$. (Table 7).

Of the sexually abused group, $14.7 \%(n=20)$ were abused in last year. About half of sexually abused group $48.55 \%(n=67)$ did not attempt to obtain assistance as, in their opinion, they were not suffering, 39.13\% $(n=54)$ did not attempt to find assistance though they were suffering and only $12.31 \%(n=17)$ attempted to find assistance. (Table 7).

The source of the sexual abuse was a known person $23.07 \%(n=27)$, others $18.8 \%(n=22)$, brother $14.52 \%$ ( $n=17)$, an unknown person, and current husband, both $12.82 \%(n=15)$. Some of the abusers were smokers $48.18 \%(n=53)$, while $4.54 \%(n=5)$ were drug abusers, and $5.45 \%(n=6)$ were abusing alcohol.

Regarding disclosure 65.18\% $(n=88)$ did not disclose the abuse, $22.22 \%(n=30)$ partially disclosed, and only $12.59 \%(n=17)$ disclosed the abuse completely. In addition, $87.5 \%(n=49)$ did not disclosed to the doctor, $1.78 \%(n=1)$ thought her doctor knew, 8.92\% ( $n=5)$ disclosed to her doctor when asked about abuse, and only $1.78 \%(n=1)$ fully disclosed to her doctor. (Table 7).

\section{Discussion}

DV is a public health issue, both nationally and internationally, and causes many adverse physical and mental health consequences. The prevalence of DV varies between countries depending on cultural taboos and the

\section{Types of Domestic Violence}

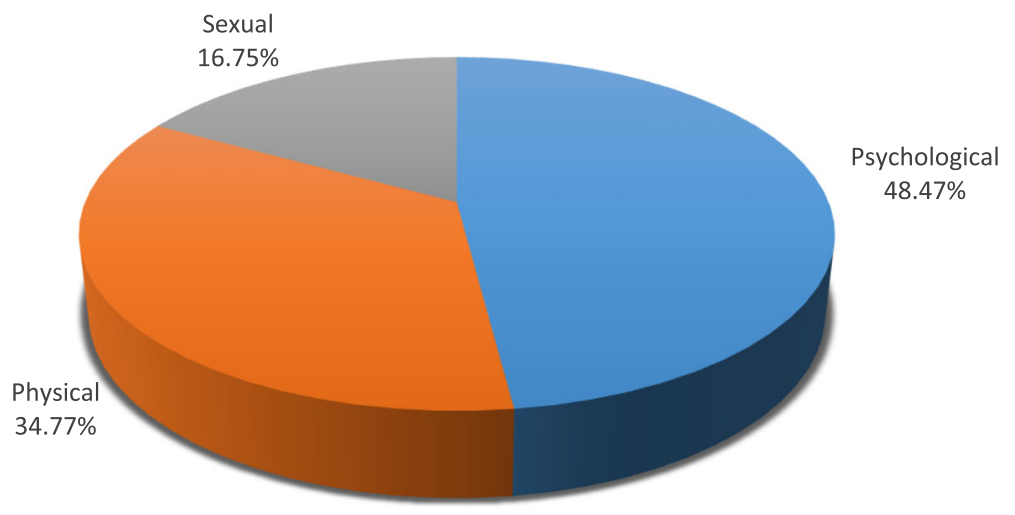

Fig. 2 Domestic Violence by Type 
Table 4 The Relationship between Demographic Characteristics and Abuse Status

\begin{tabular}{|c|c|c|c|c|c|}
\hline Variable & No & $\begin{array}{l}\text { Abuse } \\
\text { No (\%) }\end{array}$ & $\begin{array}{l}\text { No Abuse } \\
\text { No }(\%)\end{array}$ & $\begin{array}{l}{ }^{a} \text { Chi- } \\
\text { square }\end{array}$ & $p$-value \\
\hline \multicolumn{6}{|l|}{ Employment status } \\
\hline - Employed & 257 & $118(45.9)$ & $139(54.1)$ & 22.422 & .000 \\
\hline - Housewife & 1536 & 477 (31.1) & 1059 (68.9) & & \\
\hline - Retired & 4 & $2((50)$ & $2(50)$ & & \\
\hline \multicolumn{6}{|l|}{ Educational level } \\
\hline - Illiterate & 179 & $42(23.5)$ & $137(76.5)$ & & \\
\hline - Undergraduate & 822 & $252(30.7)$ & $570(69.3)$ & & \\
\hline - Graduate & 765 & $292(38.2)$ & $473(61.8)$ & 19.597 & .001 \\
\hline - Postgraduate & 31 & $12(38.7)$ & $19(61.3)$ & & \\
\hline \multicolumn{6}{|l|}{ Residence } \\
\hline - Unknown & 19 & $8(42.1)$ & $11(57.9)$ & & \\
\hline - City & 1495 & $490(32.8)$ & 1005 (67.2) & 1.773 & .635 \\
\hline - Village & 283 & $100(35.3)$ & $183(64.7)$ & & \\
\hline \multicolumn{6}{|l|}{ Home type } \\
\hline - Owned & 1074 & $367(34.2)$ & $707(65.8)$ & 1.002 & .317 \\
\hline - Rent & 721 & $230(31.9)$ & $491(68.1)$ & & \\
\hline \multicolumn{6}{|l|}{ Family income } \\
\hline • < SAR 5000 & 633 & $213(33.6)$ & $420(66.4)$ & .061 & .805 \\
\hline - > SAR 5000 & 1161 & $384(33.1)$ & $777(66.9)$ & & \\
\hline \multicolumn{6}{|l|}{ Social status } \\
\hline - Single & 404 & $158(39.1)$ & $246(60.9)$ & & \\
\hline - Married & 1295 & 394 (30.4) & 901 (69.6) & 29.330 & .000 \\
\hline - Divorce & 46 & $29(63)$ & $17(37)$ & & \\
\hline - Widow & 56 & $18(32.1)$ & $38(67.9)$ & & \\
\hline \multicolumn{6}{|l|}{ Marriage duration } \\
\hline$\bullet<5$ years & 362 & $120(33.1)$ & $242(66.9)$ & & \\
\hline - 5-10 years & 349 & $108(30.9)$ & $241(69.4)$ & 8.11 & .044 \\
\hline$\bullet \geq 10$ years & 671 & $205(30.6)$ & $466(69.4)$ & & \\
\hline \multicolumn{6}{|c|}{ Financial dependence on Alhusband } \\
\hline - Yes & 1065 & $298(28)$ & $767(72)$ & 21.191 & .000 \\
\hline - No & 309 & $129(41.7)$ & $180(58.3)$ & & \\
\hline
\end{tabular}

definition of violence. A systematic review conducted in Turkey reported that DV is prevalent in the country [15]. The estimated prevalence of DV in Arab countries, reported in a systematic review, was $73.3 \%$, with $35.6 \%$ physical, $49.8 \%$ psychological, and $22.0 \%$ sexual abuse [16].

The current study estimated the prevalence of DV in the Western Region of Saudi Arabia, including Makkah, Jeddah, and Al-Taif $33.24 \%$. The prevalence is similar to a study conducted in 2017, including 758 Saudi Arabian women from 13 governorates in Saudi Arabia, with a reported prevalence of $32 \%$ [17]. The reality, based on the
Table 5 Psychologically abused participant characteristics and source of abuse

\begin{tabular}{lll}
\hline Variable & Mean & SD \\
Age at first psychological abuse $(n=384)$ & 13.07 & 11.27 \\
Mean score of abuse consequence $(n=425)$ & 5.34 & 2.986 \\
Age of disclosure $(n=210)$ & 14.85 & 11.630 \\
Variable & Frequency & $\%$
\end{tabular}

Psychological abuse in the last year $(n=450)$

- No

- Yes

Seeking assistance $(n=451)$

- No, did not suffer

- No, although suffering

- Yes

26.60

Source of abuse $(n=602)$

- Ex-husband
- Current husband
- Stepfather
- Stepmother
- Mother
- Father
- Brother
- Sister
- Children
- Friends
- Known person
- Unknown person
- Others

What do abusers use $(n=371)$

- Smoking

- Drugs

- Alcohol

- Nothing

55.52

Disclosure

- No

- Yes, partially

- Yes, all

30.84

13.88

Disclosure to a medical practitioner $(n=230)$

- No

- Yes, she knows

2.17

- Yes, when she asked

7.39

- Yes, immediately 5.65

current and previous studies, is that at least one-third of Saudi women is a victim of abuse. The prevalence of DV in the National Guard population in the western region of Saudi Arabia is similar to the prevalence of DV 
Table 6 Physically abused participant characteristics and source of abuse

Variable
Age at first physical abuse
Mean score of physical abuse consequ
Age of disclosure
Variable
Physical abuse in the last year $(n=322$

$$
\text { - Yes }
$$$$
\text { - No }
$$

Seeking assistance $(n=323)$

- No, did not suffer

- No, although suffering

- Yes

Source of abuse $(n=228)$

- Ex-husband
- Current husband
- Stepfather
- Stepmother
- Mother
- Father
- Brother
- Sister
- Children
- Friends
- Known person
- Unknown person
- Others

Substance abuse of abusers $(n=260)$

$$
\begin{aligned}
& \text { - Smoking } \\
& \text { - Drugs } \\
& \text { - Alcohol } \\
& \text { - Nothing }
\end{aligned}
$$

Disclosure $(n=321)$

$$
\begin{aligned}
& \text { - No } \\
& \text { - Yes, partially } \\
& \text { - Yes, all }
\end{aligned}
$$

Disclosure to medical practitioner $(n=179)$

\begin{tabular}{lll} 
- No & 144 & 80.44 \\
- Yes, she knows & 3 & 1.67 \\
- Yes, when she asked & 20 & 11.17 \\
- Yes, immediately & 12 & 6.70 \\
\hline
\end{tabular}

conducted in the university hospital in the same area [17] meaning that the prevalence of DV could be consistent across different sectors regardless of the changing risk factors.

43.07

Table 7 Sexually abused participant characteristics and source of abuse

\begin{tabular}{lll}
\hline Variable & Mean & SD \\
Age at first sexual abuse & 9.92 & 7.633 \\
Mean score of sexual abuse consequence & 5.307 & 3.0282 \\
Age of disclosure & 11.92 & 8.492 \\
Variable & Frequency & $\%$ \\
Sexual abuse in the last year $(n=136)$ & & \\
- Yes & 20 & 14.7 \\
- No & 116 & 85.29 \\
Seeking assistance $(n=138)$ & & \\
- No, did not suffer & 67 & 48.55 \\
- No, although suffering & 54 & 39.13 \\
- Yes & 17 & 12.31
\end{tabular}

Source of abuse $(n=117)$

$\begin{array}{lll}\text { - Ex-husband } & 6 & 5.12 \\ \text { - Current husband } & 15 & 12.82 \\ \text { - Stepfather } & 1 & 0.85 \\ \text { - Stepmother } & 1 & 0.85 \\ \text { - Mother } & 0 & 0 \\ \text { - Father } & 5 & 4.27 \\ \text { - Brother } & 17 & 14.52 \\ \text { - Sister } & 1 & 0.85 \\ \text { - Children } & 1 & 0.85 \\ \text { - Friends } & 6 & 5.12 \\ \text { - Known Person } & 27 & 23.07 \\ \text { - Unknown Person } & 15 & 12.82 \\ \text { - Others } & 22 & 18.80\end{array}$

Substance abuse of abusers $(n=110)$

- Smoking

48.18

- Drugs

4.54

- Alcohol

5.45

- Nothing

41.81

Disclosure $(n=135)$

$\begin{array}{lll}\text { - No } & 88 & 65.18 \\ \text { - Yes, partially } & 30 & 22.22 \\ \text { - Yes, all } & 17 & 12.59\end{array}$

Disclosure to a medical practitioner $(n=56)$

- No

- Yes, she knows

- Yes, when she asked

8.92

- Yes, immediately

1.78

The current study estimated the prevalence of psychological (48.47\%), physical (34.77\%), and sexual abuse (16.75\%). Compared with studies done in Saudi Arabia, sexual abuse had a lower prevalence, but physical and 
psychological abuse was more prevalent [10-12]. The study indicated that women who experience suffering due to the abuse, had negative physical or mental health consequences, adversely affecting the victim's life [18].

Specific socio-demographic characteristics are associated with an increased or decreased risk of DV. For example, in terms of educational level, in a study done in West Bengal, the highest prevalence of DV was in the illiterate group (46.15\%) [19]. A study conducted in Sevas, Turkey, also reported the highest prevalence of DV in the illiterate group [20]. In contrast, the current study concluded that the postgraduate education group had the highest prevalence of abuse, as also reported in the study conducted in Riyadh [21]. Possible explanations could be that the higher education level is associated with financial independence, a higher awareness of DV, and the different types of abuse, enabling women to consider particular behavior as acceptable or tolerable. Besides, the abuser could feel threatened and unsafe with educated women. Employed women also had a higher prevalence of abuse compared to housewives which are similar to another study conducted in Jeddah during the year 2002 [12], which could be due to the same reasons. Lastly, the difference could be cultural and the availability of a reporting system in both countries. No studies, according to the researcher's knowledge, explained this hypothesis.

Another socio-demographic factor, financial dependence on a husband, reduced the risk of DV compared to financially independent women. In contrast, a study done in Jeddah reported that women, who were financially dependent on a husband, had 1.5-fold odds of being physically abused by the husband [12].

In the current study, women were abused at a young age, but they did not report the abuse to another person until several years later. It is noteworthy that more than half of the victims of psychological and physical abuse were still being abused in the last year, while sexual abuse tends to decrease as the woman matures.

All most all of the victims of all types of abuse are reluctant to seek assistance $97.2 \%$. Possible reasons could be due to being in a conservative culture and fear of shame, normalizing some of the acts of abuse in a predominantly male culture, or because they do not know where to find assistance. The results of the study emphasize the need to create awareness, teach women how to say no for abuse, and better understand the concept of abuse and screening programs. Another important aspect is the role of social stigma, which may have rendered the participants who were interviewed, mostly the illiterate and undergraduate groups, fearful of exposing such information. A study conducted in the USA reported that abused women are reluctant to report being abused due to feeling unsafe, fear of more severe abuse if the abuser found out, or an unclear reporting pathway [22].
In both psychological and physical abuse, the abuser was the husband or ex-husband if the victim was married and the father or brother if she was not married. Sexual abuse was perpetrated by the brother or a person known to the family. According to the Center for Disease Control and Prevention, risk factors for being abused are witnessing DV as a child, living in a controlling relationship, low income, emotional and financial dependency, and being abused as a child [23].

From the current study, abusive behaviors were common among substance abusers, with smoking most prevalent, followed by drugs and alcohol. The finding is similar to a study conducted in India, where abusers are commonly found to be substance abusers [24]. The findings support the development of a drug-screening program to decrease the incidence of DV.

\section{Study limitations}

There may be some possible limitations in this study. For example, sampling bias, where the selected population may not represent the real population under study which can affect the generalizability of the results. The sensitivity of the questions in the questionnaire is another limitation because it addresses sensitive issues like sexual abuse. Being afraid of stigma or labeling was another issue, although it was addressed in the consent form, some participants may still be hesitant to answer. Not only that, some questions were not answered thoroughly, which change the total number of responders from one question to another.

To overcome this limitation in the future, this research can be conducted on another population to help in generalizing the results. Second, using an online questionnaire may help to have more responders and complete answers.

\section{Implications for practice and research}

This study showed that Domestic violence is prevalent in Saudi Arabia, like other parts of the world. Besides, it showed that risk factors for being abused are changing. With this new information, stakeholders need to address those factors by health education and screening programs.

This study also showed that women would not volunteer the information about being abused until she was asked by a health professional, which emphasizes the need for screening programs.

It is the first Saudi study that shows a shift in the risk factors for domestic violence; the same study should be conducted on different populations to confirm the findings.

\section{Conclusion}

$\mathrm{DV}$, in all its forms, is prevalent in the women attending the National Guard Primary Health Care Clinics, as well as globally. With the rapid social openness in the form of allowing women to drive cars, to be independent after 
the age of 18 and full custody of her children, and much more, there is a shift in the risk factors for being a victim of DV. Culture-specific preventive and screening programs can contribute to reducing contributing factors for perpetrating abuse, supporting a reduction in the prevalence of abuse in the Saudi community.

\section{Abbreviations}

DV: Domestic violence; KAMC: King Abdulaziz Medical City; NORAQ: Norvold Domestic Abuse Questionnaire; WHO: World Health Organization

\section{Acknowledgments}

We want to thank all women who participated in this study and data collectors (Bayader Shami, Razan Alharbi, Danah Bakhallagi, Haneen Falata, Esraa Alfayoumi, Amal Almutairi, Hadeel Guhal, Raghad AlQurashi, Amal Alnemare, Bashaier AlQahtani, Asayel Alwthenani, Duaa Sabri, and Omnia Wawi).

For their contribution to the success of this research and dr Altaf Khan, who helped with the data analysis.

\section{Authors' contributions}

All Authors contributed to all the parts, proposal writing, data collection, data analysis, and writing the discussion. The correspondence author (RW) is the owner of the intellectual idea, analysis, writing the result, and the discussion section and final review. AK contributed to the proposal and method section writing and data collection and entry. RA contributed to writing the proposal, background, data collection, data entry, and wrote some of the discussion points. RF contributed to data collection, writing the background section analysis and writing part of the discussion. IM contributed to data collection, analysis, and writing the results. SS contributed to proposal writing, method section, and data collection. All authors read and approved the final manuscript

\section{Funding}

This research is self-funded.

\section{Availability of data and materials}

All data generated or analysed during this study are included in this published article.

\section{Ethics approval and consent to participate}

Ethical approval was obtained from the Institutional Review Board of King Abdullah International Medical Research Center with a reference number. SP18-012-J. Ethical principles were maintained throughout the research process. All participants signed informed consent, and confidentiality and anonymity assured as no personal identifiers were used. All data were stored on workplace computers with access to study personal only.

\section{Consent for publication}

Written informed consent for publication was obtained.

\section{Competing interests}

The authors declare that they have no competing interests.

\section{Author details}

${ }^{1}$ Ministry of National Guard-Health Affairs, Jeddah, Saudi Arabia. ${ }^{2}$ King Abdullah International Medical Research Center, Jeddah, Saudi Arabia. ${ }^{3}$ King Saud Bin Abdulaziz University For Health Sciences, Jeddah, Saudi Arabia.

Received: 7 October 2019 Accepted: 3 January 2020

Published online: 17 February 2020

\section{References}

1. What is Domestic Violence? [Internet]. Ncadv.org. 2017 [cited 13 April 2017]. Available from: http://ncadv.org/learn-more/what-is-domestic-violence .

2. What is domestic violence? - Domestic Violence [Internet]. Domesticviolence.nsw.gov.au. 2017 [cited 14 April 2017]. Available from: http://www.domesticviolence.nsw.gov.au/what-is-domestic-violence.

3. Fink G. Stress consequences: mental, neuropsychological and socioeconomic. 1st ed. Amsterdam: Elsevier Academic Press; 2010.
4. Al Dosary AH. Health impact of domestic violence against Saudi women: cross sectional study. Int J Health Sci. 2016;10(2):165-73.

5. Responding to intimate partner violence and sexual violence against women. WHO clinical and policy guidelines. https://www.who.int/ reproductivehealth/publications/violence/NAW_Prevelance.jpeg?ua=1

6. Yount KM. Resources, family organization, and domestic violence against married women in Minya, Egypt. J Marriage Fam. 2005;67(3):579-96.

7. Douki S, Nacef F, Belhadj A, Bouasker A, Ghachem R. Violence against women in Arab and Islamic countries. Arch womens Ment Health. 2003:6(3):165-71.

8. Indu PV, Remadevi S, Vidhukumar K, Shah Navas PM, Anilkumar TV, Subha N. Domestic Violence as a Risk Factor for Attempted Suicide in Married Women [published online ahead of print, 2017 Aug 1]. J Interpers Violence. 2017:886260517721896. https://doi.org/10.1177/0886260517721896.

9. Newswire F. Saudi Women Continue Finding Ways to Flee Violence: Feminist Daily Newswire; 2019. [Cited 3 July 2019]. Available from: https:// feminist.org/blog/index.php/2019/04/19/saudi-women-continue-findingways-to-flee-violence/

10. Tashkandi A, Rasheed FP. Wife abuse: a hidden problem. A study among Saudi women attending PHC centres. East Mediterr Health J. 2009;15:1242-53.

11. Afifi ZE, Al-Muhaideb NS, Hadish NF, et al. Domestic violence and its impact on married women's health in eastern Saudi Arabia. Saudi Med J. 2011;32:612-20.

12. Fageeh W. Factors associated with domestic violence: a cross-sectional survey among women in Jeddah, Saudi Arabia. Br Med J Open. 2014;4(2):e004242

13. Alquaiz AM, Almuneef M, Kazi A, Almeneessier A. Social determinants of domestic violence among Saudi married women in Riyadh, Kingdom of Saudi Arabia. J Interpers Violence. 2017. https://doi.org/10.1177/0886260517746128.

14. Haddad LG, Shotar A, Younger JB, Alzyoud S, Bouhaidar CM. Screening for domestic violence in Jordan: validation of an Arabic version of domestic violence against women questionnaire. Int J Women's Health. 2011;3:79.

15. Özcan NK, Günaydın S, Çitil ET. Domestic violence against women in Turkey: A systematic review and Meta analysis. Arch Psychiatr Nurs. 2016;30(5):620-9.

16. Hawcroft C, Hughes R, Shaheen A, Usta J, Elkadi H, Dalton T, et al. Prevalence and health outcomes of domestic violence amongst clinical populations in Arab countries: a systematic review and meta-analysis. BMC Public Health. 2019:19(1):315.

17. Halawi A, Almalki Z, Ahmed A. Prevalence and risk factors for abuse among Saudi females, KSA. Egypt J Hosp Med. 2017:68(1):1082-7.

18. Sugg N. Intimate partner violence: prevalence, health consequences, and intervention. Med Clin N Am. 2015:99(3):629-49.

19. Sarkar M. A study on domestic violence against adult and adolescent females in a rural area of West Bengal. Indian J Community Med. 2010;35(2):311-5.

20. Kocacik F, Dogan O. Domestic violence against women in Sivas, Turkey: survey study. Croat Med J. 2006;47(5):742-9.

21. Barnawi FH. Prevalence and risk factors of domestic violence against women attending a primary Care Center in Riyadh, Saudi Arabia. J Interpers Violence. 2017:32(8):1171-86.

22. Gielen AC, O'Campo PJ, Campbell JC, Schollenberger J, Woods AB, Jones AS, et al. Women's opinions about domestic violence screening and mandatory reporting. Am J Prev Med. 2000;19(4):279-85.

23. Center for Disease Control and Prevention (CDC). Risk and Protective Factors for Perpetration https://www.cdc.gov/violenceprevention/ intimatepartnerviolence/riskprotectivefactors.html

24. Bhatt RV. Domestic violence and substance abuse. Int J Gynecol Obstet. 1998;63(S1):S25-31.

\section{Publisher's Note}

Springer Nature remains neutral with regard to jurisdictional claims in published maps and institutional affiliations. 